\title{
30. RADIAL VELOCITIES (VITESSES RADIALES)
}

\author{
PRESIDENT : G. Burki \\ VICE-PRESIDENT: C.D. Scarfe \\ ORGANIZING COMMITTEE : $\quad$ J. Andersen, L.N. da Costa, A.P. Fairall, \\ K.C. Freeman, J.B. Hearnshaw, D.W. Latham
}

\section{RADIAL VELOCITIES OF GALAXIES (A.P. Fairall, Cape Town University)}

Cosmological implications, arising from the existence of large-scale structures and large-scale flows, have been major motivations for redshift surveys of galaxies and clusters of galaxies. This has been coupled with technical advances in the sensitivity of CCD detectors and multiple-object observations using fibres. Many fibre systems are now producing a hundred or more radial velocities of faint galaxies per night. Amongst current developments is the MEFOS system at the European Southern Observatory (where fibres are positioned via computer control, and which will supersede the older "plug in" OP. TOPUS system). Also, at the Anglo-Australian Observatory, a system is under development that will enable 400 fibres (set in position by computer) to cover a two degree field on the $3.9 \mathrm{~m}$ telescope, while a new Flair II (152 fibres) system has been commissioned on the wide-angle Schmidt telescope.

Although many investigators are publishing results and analyses of their redshift surveys (and showing plots of redshift "slices" at conferences), relatively few of the radial velocities are actually appearing in the literature. In part, this may be that the radial-velocity databases are intentionally being kept confidential for the time being. However, it may also indicate problems in mass reductions - since there are some fibre-redshift papers that show a greater proportion of discrepant redshifts than was the previous norm. A rough guess is that there are now "public" redshifts for approximately 30000 galaxies, with a similar number as yet unpublished.

Wide-angle surveys are not normally able to make use of fibres. The extension of the Centre for Astrophysics redshift survey (CfA2) to $m=15.5$ is complete over twelve declination slices. In the South, the complementary extension of the Southern Sky Redshift Survey (SSRS2) by L. da Costa \& collaborators is virtually complete. M. Strauss \& collaborators have published radial velocities for an all-sky survey of selected IRAS galaxies. The QDOT survey of Ellis, Rowan-Robinson \& collaborators covers a similar sample.

Using fibres, the Las Campanas Deep Redshift Survey is coverering both a "Checkerboard" pattern of areas and declination strips in the southern skies, and has over 10000 redshifts. A "key-project" is under way at ESO by Vettolani \& collaborators, using multi-fibres, that should obtain some 5000 redshifts of galaxies with $\mathrm{B}(\mathrm{J})<19.5$ in a strip of Declination 1.5 degrees wide. Koo \& collaborators are continuing their deep pencil-beam surveys that revealed unexpected periodicity in distribution of redshifts in the directions of the galactic poles. A number of groups are looking at Abell and AbellCorwin-Olowin clusters, such that the overlaps should in time provide a good comparison of radial velocity measurements. Kraan-Korteweg \& collaborators have a large number of redshifts for galaxies partly obscured by the southern Milky Way.

The recent Principal Galaxy Catalogue (Paturel et al., 50.002.016) and the 3rd Reference Catalogue (de Vaucouleurs el al., 53.002.034) serve as useful sources of galaxy radial velocities. An ongoing database of galaxy redshifts (ZCAT) is maintained at the Center for Astrophysics by J. Huchra \& collaborators, and an independent one for southern galaxies (SRC) is kept by A. Fairall in Cape Town. Redshifts can also be obtained through IPAC (NASA extragalactic database) and the Astronomical Data Centres at Goddard and Strasbourg. In respect of cataloguing, an appeal is made to all observers to publish accurate positions, and not just designations of galaxies, and to publish heliocentric velocities. Otherwise much of their efforts may be lost to the astronomical community.

\section{STARS IN THE LOCAL GROUP OF GALAXIES}

(K.C. Freeman, Mount Stromlo Observatory)

2.1. Magellanic Clouds.

IAU Symposium 148 (53.012.005) includes papers on velocities of Magellanic Cloud objects. Cowley \& Hartwick (53.156.148) measured velocities for $40 \mathrm{CH}$ stars in the LMC; these stars have an asymmetric velocity distribution suggesting two discrete kinematical groups. Hughes et al. (53.156.079) studied the kinematics of the long period variables in the LMC; the oldest 
stars indicate the presence of a spheroidal population in the LMC. Vassiliadis et al. (1992) measured velocities of planetary nebulae in the outer LMC.

Hatzidimitriou et al. (1993) showed that the radial velocities of clump giants in the outer SMC are well correlated with the distance along the line of sight, over a line of sight distance of at least $10 \mathrm{kpc}$; this may be the result of tidal interaction of the SMC and LMC.

The kinematics of star clusters in the LMC were actively studied. Storm et al. (53.156.137) measured velocities of 3 distant LMC clusters. Schommer et al. (55.156.003) observed giants in 83 clusters at the Ca triplet and confirmed that the oldest clusters appear to lie in a rotating disk. Suntzeff et al. (1992) measured velocities for NGC 1841 and the Reticulum cluster, confirming their membership in the LMC and estimating their $\mathrm{M} / \mathrm{L}$ ratios from the internal velocity dispersion. Fischer et al. (1993) observed 52 supergiants in the young LMC binary cluster NGC 1850 to estimate its M/L ratio.

2.2. Dwarf Spheroidal Galaxies.

Pryor \& Kormendy (52.157.024) reanalysed the kinematics of the Draco and UMi galaxies, and showed that their central dark matter densities are at least 0.05 $M_{\odot} p c^{-3}$ but could be as high as $1 M_{\odot} p c^{-3}$. Da Costa et al. (53.157.225) measured radial velocities of 14 giants in the Sextans galaxy, and derived a systemic velocity of $230 \mathrm{kms}^{-1}$. Mateo et al. (54.157.198) made a major study of the Fornax galaxy, with new velocities for 44 giants and 4 globular clusters; they derived a central M/L ratio of 12 . Mateo et al. (1993) measured velocities for 23 giants in the Carina galaxy; the velocity dispersion is $6.8 \mathrm{kms}^{-1}$ and the M/L ratio is 39 , so Carina's dark matter content is intermediate between Draco and UMi on one hand and the larger dwarf spheroidal galaxies on the other.

Munari (54.157.147) studied a symbiotic carbon star in the Draco galaxy. This star shows significant radial velocity variations and appears to be a binary (carbon giant with a hot compact companion).

2.3. M31. Federici et al. $(52.157 .044,54.154 .093,55.157 .305)$ studied the velocities of globular clusters in M31 to estimate the mass of M31 and investigate the orbital properties of the cluster system. The data suggest that the clusters have circular or isotropic orbit distributions. Huchra et al. (53.154.028) measured velocities and metallicities for a large sample of clusters in M31. They find that the inner metal rich clusters form a central rotating disk, but in the outer regions there is no distinction between the rotation of the metal rich and metal poor clusters.

2.4. M33. Schommer et al. (53.157.024) measured the kinematics of star clusters in M33. The younger clusters rotate with the HI disk, while the older clusters form a slowly rotating halo.

\section{STRUCTURE OF THE MILKY WAY (D.W. Latham, Center for Astrophysics, and}

B.W. Carney, University of North Carolina)

Radial velocities have been used to study several aspects of the Galactic center, which has a diversity of structures that may even include a black hole. The gas near the center has been studied by Mehringer et al. (1992) in the vicinity of Sgr B1 and B2 . Herbst et al. (1993) have used infrared imaging spectroscopy to obtain high spatial (1") and low velocity $(100 \mathrm{~km} / \mathrm{sec})$ resolution near the center. Binney et al. (54.155.109) have discussed the issues involved in the studies of gas dynamics of the center. Stars are immune to hydrodynamical effects, and new radial velocity work on $\mathrm{OH} / \mathrm{IR}$ stars has been undertaken by Lindqvist et al. (55.155.096) and by Langevelde (Leiden) \& his colleagues. Work is continuing on high-resolution IR spectroscopy of M giants by Sellgren (Ohio State) \& the Riekes (Arizona). Blitz \& Spergel (54.155.089) conclude that the H I kinematics in the Galaxy's central regions are best understood if there is a bar, following the earlier suggestions of Binney et al. (54.155.109).

The dynamics of the Galaxy's central bulge have also received considerable attention. Minniti et al. (1992) have studied the kinematics of $\mathrm{K}$ giants in two bulge fields, finding strong evidence for rotation and a velocity dispersion that decreases with increasing galactocentric distance. The transition between the bulge and the galactic halo is being studied by Morrison (1993), using objective prism techniques to identify $\mathrm{K}$ giants and radial velocities to help assign the stars to their respective stellar populations. The bulge or the thick disk appear to dominate, even at distances that used to be considered the province of the halo.

The study of stellar populations in the Galaxy has benefitted tremendously from extensive radial velocity programs. A survey of proper motion stars was completed by Norris \& Ryan (54.155.095), who suggest that the thick disk may not be a discrete component but is instead an older, hotter extension of the thin disk. A survey of the kinematics and metallicity of an expanded survey of proper-motion stars by B. Carney (North Carolina), D. Latham (CfA), and J. Laird (Bowling Green) is nearing completion, as 
are surveys of southern hemisphere proper motion stars by Ardeberg et al. (53.115.046) and M. Grenon (Geneva). Kinematically unbiased samples are receiving attention, too, especially the south galactic pole K giants studied by Flynn \& Freeman (1993), faint proper motion stars toward the north galactic pole studied by Majewsli (55.155.008), and the faint stars in the galactic meridional plane being studied by R. Wyse (Johns Hopkins) \& G. Gilmore (Cambridge). Radial velocities from these programs should help us understand the relative numbers and evolutionary histories of the major stellar populations.

The fundamental parameters describing galactic structure are becoming better defined from kinematical studies. Jaschek \& Valbousquet (53.155.035) have observed 5800 nearby bright stars to study the Sun's motion and stellar velocity dispersions as a function of spectral type, while Kuijken \& Gilmore (53.155.022) have discussed the Galactic force law and the disk's column mass density.

The Galaxy's total mass, based on radial velocities of distant stars and satellite systems, was discussed by Norris \& Hawkins (54.155.093), and by Kulessa \& Lynden-Bell (55.155.090), with the conclusion that the Galaxy's total mass exceeds $1.210^{12} M_{\odot}$.

\section{STAR CLUSTERS (J.C. Mermilliod, Université de Lausanne)}

4.1. Open clusters. The interest for the measurement of radial velocities has received new motivation due to the new instruments and detectors and the generalisation of the correlation technique. The results obtained are encouraging: it seems possible to obtain radial velocities with a good precision, 2 to $3 \mathrm{~km} / \mathrm{s}$, for early type stars. This has been demonstrated with observations of stars in the Pleiades by two different groups (Morse et al., 53.036.025; Liu et al., 54.111.004) and in other young open clusters (Liu et al., 54.153.056).

Old favorites have been revisited: Alpha Persei and IC 4665 (Morrell \& Abt, 54.153.037; 1992). The situation in IC 4665 is even more confused, because further binaries have been rejected from binarity: only four of the 15 stars observed are spectroscopic binaries. The detection of binaries on the upper main sequence has been extended to the Orion cluster (Abt el al., 53.153.15) and to two clusters located in the Eta Carinae region, $\operatorname{Tr} 14$ and $\operatorname{Tr} 16$ (Levato et al., 54.153.016; 53.153.026).

Photoelectric scanners have been used to measure radial velocities of red dwarfs in nearby open clusters to search for binaries in Praesepe (Bolte, 54.153.017) and in the Pleiades (Mermilliod et al., 1992), to identify new member stars in the halo of the Pleiades (Rosvick et al., 55.153.002) and Praesepe clusters (Mermilliod et al., 52.153.006). Radial velocities have been obtained to confirm the membership of low-mass stars in M67 (Glushkova \& Rastorguev, 53.153.022), Pleiades (Soderblom et al., 1993), Alpha Persei cluster and IC 4665. (Prosser, 55.153.010; 1993). The most remarkable result is the publication of the orbital elements for 22 spectroscopic binaries among red giants and upper main sequence stars in M67 (Mathieu et al., 52.153.020).

Red giants in numerous clusters have also been observed with photoelectric scanners (Mermilliod \& Mayor, 52.153.009; Valitova et al., 52.153.43; Glushkova \& Rastorguev, 53.153.022, 53.153.035, 53.153.036; Claria \& Mermilliod, 1992). The aims of most of these studies are to determine the membership of the red giants, to discover new binaries or to define the global cluster velocity.

The cross-correlation technique was also used to obtain radial velocities and metallicity determinations for red giants in several old open clusters (Friel \& Janes, 1993).

4.2. Globular clusters.

Photoelectric scanner radial velocities have been obtained for 159 stars in M4, M5, M10, M12 and M71 (Ortolani et al., 54.154.127) to study the velocity dispersion. Meylan \& Mayor (54.154.029) have used radial velocities for 127 stars in NGC 6397 in order to calculate the velocity dispersion and to compute a multi-mass, anisotropic dynamical (King-Michie) model from which the cluster characteristics have been determined. On the basis of radial velocities for 72 stars in the chusters NGC 5053 and 5466, Pryor et al. (54.154.105) have shown that five of these stars are probably spectroscopic binaries.

4.3. Magellanic Clouds clusters. Radial velocities with a precision of about $1 \mathrm{~km} / \mathrm{s}$ have been obtained in NGC 1866 (Fischer et al., 55.154.015). The observed flattening of the cluster is consistent with the rotation deduced from the radial velocities.

\section{SPECTROSCOPIC BINARIES}

(A. Duquennoy and M. Mayor, Observatoire de Genève)

Of course the domain of spectroscopic binaries is still a very rich, active and fruitful domain of astronomy. This can be seen from an inspection of the section 120 of the $A$ \& $A$ Abstracts which lists 
each year more than 100 entries for spectroscopic binaries. Evidently one can only select here a few references, which we hope will well illustrate the rich and exciting new results and developments in the field of spectroscopic binaries.

The various leading groups, in terms of high precision velocities, are still the same as in the 1988 IAU report. Reaching a long-term precision better than $50 \mathrm{~m} \mathrm{~s}^{-1}$ will be soon a common performance. At this level of precision one has difficulties to distinguish between velocity variations due to a companion and those due to intrinsic instabilities inside the star. Such high-precision radial-velocity surveys are in progress at McDonald, CFHT and Lick Observatories in order to detect planetary sized companions.

An important novelty in the domain of the SBs is the incursion of high angular resolution techniques, now capable to resolve SBs with periods of only a few tens of days and semi-major axis of the order of 0.01", thanks to long base interferometry: MARK III at Mt Wilson, USA, and next generation SUSI, in Australia (see IAU Coll. No 135 on Complementary Approaches to Double and Multiple Star Research, 1992, San Francisco), and thus opening the way to "massive" production of stellar masses determinations.

Spectroscopic binaries continue to be accumulated among various stellar populations: Pre-MainSequence stars (Andersen, 52.120.007; Mathieu et al., 53.120.011; Pasquini et al., 54.117.041), WolfRayet stars (Williams \& van der Hucht, 52.120.023; Annuk, 53.117.011), IAU velocity standards (Scarfe, 55.120.022), Post-AGB stars (Griffin, 53.120.016), White Dwarfs (Brabaglia et al., 52.126.074), F str $\lambda 4077$ stars (North \& Duquennoy, 53.113.010) and other peculiar stars (Howarth \& Heber, 52.120.012; Morrell \& Niemela, 52.120.022).

R. F. Griffin continues to produce spectroscopic orbits for a number of stars including composite spectra (Griffin, 52.120.019, 55.120.023), eclipsing binaries (Griffin et al., 55.119.001), long-P SBs, $P>$ $10 y r s$ (Griffin \& Herbig, 54.120.007), some of them having low-K amplitudes, $K_{1}<2 \mathrm{~km} \mathrm{~s}^{-1}$ (Griffin, 53.120.016). At this point, it seems strange that with the increasing precision of the radial velocity determinations, no very low amplitude $\left(K_{1}<0.1 \mathrm{~km} \mathrm{~s}^{-1}\right)$ binaries have yet been found. This probably means that brown dwarf companions are rare if any.

However predictions of the rate of brown dwarf companions can be made from systematic surveys for duplicity in unbiased samples. In the solar neighbourhood, this proportion is estimated to $8 \pm 6 \%$ of the G-dwarf primaries (Duquennoy \& Mayor, 54.118.010).

Other recent systematic and fundamental spectroscopic surveys for duplicity include: nearby stars (Tokovinin, 54.120.026; Fischer \& Marcy, 1992), halo stars (Latham et al., 1992), S stars (Jorissen \& Mayor, 1992), open clusters (Mermilliod et al., 52.153.006; Mermilliod \& Mayor, 52.153.009; Mathieu et al., 52.153.020; Mermilliod et al., 1992), globular clusters (Prior \& al., 54.154.105). They mainly tend to obtain unbiased distributions of orbital elements, especially of Period, Eccentricity and Mass-Ratios, which are believed to be tracers of the binary star formation processes.

The importance of these systematic surveys is considerable, as they allow unprecedented insights into the (sometimes combined) effects of physical processes among SBs, such as tidal effects, AMLOSC, wind accretion, dynamical evolution, overshooting in stellar evolution model, and of course star formation mechanisms. This was the subject of a workshop held in Switzerland to honour R.F. Griffn's 100th paper in The Observatory. Twenty-three contributions were included in the proceedings $(1992$, in Binaries as Tracers of Stellar Formation, Cambridge).

\section{PUlsating Stars (G. Burki, Observatoire de Genève)}

The Baade-Wesselink method, requiring quasi-simultaneous photometric and radial velocity measurements of pulsating stars, allows the determination of mean radius and distance (if the effective temperature is also known) of the studied objects. Because of the increasing performances of the radial velocity spectrometers, it is now possible to derive high quality velocity curves for several tens of field RR Lyrae stars and for some of these stars in globular clusters.

The absolute magnitude of field RR Lyrae has been determined for SW And and DX Del by Jones et al. (55.122.027), for UU Cet, RV Phe and W Tuc by Cacciari et al. (1992) and for V445 Oph, SS Leo and VY Ser by Fernley et al. (55.122.077). Moreover, respectively 4, 2 and 2 RR Lyrae have been analysed in the globular clusters M4 (Liu \& Janes, 52.122.022), M5 and M92 (Storm et al., 55.122.015). The impact of these determinations on the luminosity-metallicity relation of RR Lyrae stars has been discussed by Carney et al. (55.122.028).

The Baade-Wesselink method has also been applied to the cepheid stars of the remarkable young globular cluster NGC 1866 in the LMC (Welch et al., 53.122.001; Côté et al., 53.122.012). This is a unique case of a stellar cluster containing a lot of cepheids, allowing a very powerful test of the Baade-Wesselink method.

The distances to 101 cepheids were determined by Barnes et al. (53.122.163), by using the surface 
brightness version of the Baade-Wesselink method. An independant solution for the Period-Luminosity relation of the cepheids was then established.

The mean radial velocity ( $\gamma$ velocity) of 58 cepheids has been derived by Metzger el al. (53.111.010, 55.111.005); these new values have been used to improve the definition of the rotation curve of the Galaxy. Similarly, Fernley et al. (1993) got new radial velocity data for a sample of RR Lyrae stars in order to derive the mean absolute magnitude of these stars by means of the statistical parallaxes method.

The atmospheric motions in various types of pulsating stars have been analysed by using simultaneous radial velocity and high-resolution spectroscopic observations. A series of papers have been published by D. Gillet \& collaborators on the RV Tauri stars AC Her and R Scu (Gillet et al., 52.122.012), on $\beta$ Cephei stars $\beta$ Cep, $\sigma$ Sco and 12 Lac (Mathias et al., 54.122.063, 55.122.055) and on W Vir (Lèbre \& Gillet, 55.122.009).

\section{IAU STANDARD STARS}

(R.P. Stefanik, Center for Astrophysics, and

C.D. Scarfe, University of Victoria)

A meeting of the Working Group on Radial Velocity Standard Stars took place during the IAU General Assembly XXI. The results of that meeting, and velocity information on standard stars and candidates for standard stars, can be found in the Report of Commission 30: Radial Velocities, in Reports on Astronomy, IAU Transactions XXIB, 1992. The goal of the Working Group is to provide a list of standard stars whose velocities are known with an accuracy of $100 \mathrm{~m} / \mathrm{s}$. Problems requiring further attention include a color-dependence in the comparison of velocities from several observatories, velocity variables among the standard stars, and extending the list of standards to cover a wider range of spectral and luminosity classes.

\subsection{Early-type velocity candidates.}

F. Fekel continues to observe about 35 early-type stars as possible radial velocity standards (see the list in IAU Transactions XXIB, 1992). Most stars remain good candidates, but some show possible velocity variations. Two excellent candidates are HD 27962 (Cole et al., 55.118.008) and HD 128167 (Hartkopf et al., 55.118.013), while HD 147394 should be dropped because it shows asymmetries in its line profiles. Fekel suggests adding HD 48843 to the list.

J. Morse \& R. Mathieu report that the Fekel list ties in well with the Pleiades and Alpha Persei velocities derived from late type stars (Morse et al., 53.036.025).

As part of an effort to use HIPPARCOS proper motions to set space motions and to study the dynamics of young stellar groups, W. Verschueren continues work on obtaining accurate radial velocities of early-type stars, including standards, from echelle spectra. Some results of this effort can be found in his $\mathrm{PhD}$ Thesis (1991, Free University of Brussels).

\subsection{Late-type velocity candidates.}

7.2.1 DAO work. At DAO, observations of IAU standards have been continued photoelectrically by R. McClure \& C. Scarfe, and photographically by Scarfe \& A. Batten. To search for colordependencies, Scarfe has observed most of the northern standards photoelectrically with each of two masks, based on the spectra of Arcturus and Procyon. No color-dependent difference has been found between the masks, or between them and the photographic data in Scarfe et al. (51.111.008).

7.2.2. CfA Standard Program.

The CfA team (Latham, 1992) continues to monitor IAU radial velocity standard stars, with nearly ten years of coverage and over 5000 observations with a precision of 0.3 to $0.4 \mathrm{~km} / \mathrm{sec}$. In addition, CfA monitors a number of stars which are candidates for radial velocity standards: a) 18 solar-type stars found to be constant by the CORAVEL team, with over $400 \mathrm{CfA}$ observations and more than 500 days' coverage; and b) eight stars found to be constant by the Canadian HF-cell precise-velocity team, with over $130 \mathrm{CfA}$ velocities and more than 500 days' coverage. Also being monitored are several CfA cluster standards and four Griffin (1969) standards. Lists of the candidate stars can be found in IAU Transactions XXIB, 1992. CfA also continues to observe minor planets, with more than 700 observations, in order to establish an absolute zero-point for the CfA system. In order to optimize the match between the observed star spectra and the cross-correlation templates, CfA has started using calculated, synthetic spectra for the templates. The synthetic spectra are based on Kurucz model atmospheres and cover a dense grid of effective temperature, surface gravity, metallicity and rotational velocity. 
7.2.3. DAO-CfA comparison.

The zero-point difference between Scarfe's combined DAO photoelectric data and the CfA system is independent of color, and has been used to shift the former to the latter's zero point. There is no difference in scale between the systems. The combined mean data do not confirm the existence of a difference in zero-point between the bright and faint groups of standards. However, the differences between them and the mean velocities from CORAVEL are correlated with the stars' color indices, becoming increasingly negative for redder stars.

Several apparently variable stars have been identified, and excluded from these comparisons. HD 156014 and HD 115521 are confirmed as low-amplitude variables; the latter is possibly triple. Among the Heard-Fehrenbach objects, in addition to HD 42397 (Scarfe, 55.121.022), HD 140913 and HD 171232 appear to be variable, the latter on a long time scale. A preliminary orbital solution for HD 140913 suggests some similarity to HD 114762 (Latham et al., 49.118.004).

7.2.4. The CORAVEL Standard Program.

The CORAVEL team (Duquennoy \& Mayor, 54.118.010) has been monitoring more than 100 standard stars for more than 12 years and have about 8000 velocity measurements with a precision of 0.2 to $0.3 \mathrm{~km} / \mathrm{sec}$. Several velocity variables have been found; those having orbital solutions are HD 3346, HD 35410, HD 44131, HD 115521 and HD 123782. In addition, they have been monitoring solar-type stars for more than a decade. Potential candidates drawn from this sample can be found listed in IAU Transactions XXIB, 1992.

7.2.5. Mt. John work.

J. Hearnshaw \& his students have been actively observing IAU standards from Mt. John University Observatory, New Zealand. Using a photoelectric velocity scanner and oscillating mask on a fiber-fed echelle spectrograph, LUCIFER, they have made about 1500 observations of 30 IAU standards. A precision of about $100 \mathrm{~m} / \mathrm{s}$ has been achieved.

$\mathrm{K}$. Murdoch \& colleagues, using cross-correlation techniques and the fiber-fed echelle, have a program for obtaining radial velocities to a precision of $50 \mathrm{~m} / \mathrm{s}$. Among the stars being monitored are 12 IAU standards. After 2.5 years of observations five standards show evidence of low-amplitude variations: HD 18884, HD 81797, HD 108903, HD 146051, HD 204867 (Murdoch \& Hearnshaw, 54.111.011; Murdoch et al., 55.111.007, 1993).

\section{REFERENCES}

Cacciari, C., Clementini, G., Fernley, J.A., 1992, Ap.J. 396, 219.

Claria, J.J., Mermilliod, J.-C., 1992, A.A. Sup. 05, 429.

Fernley, J.A., Skillen, I., Burki, G., 1993, A.A. Sup. 97, 815.

Fischer, D.A., Marcy, G.W., 1992, Ap.J. 396, 178.

Fischer, P., Welch, D.L., Mateo, M., 1993, A.J. $105,938$.

Flynn, C., Freeman, K.C., 1993, A.A. Sup. 97, 835.

Friel, E.D., Janes, K.A., 1993 A.A. 267, 75

Griffin, R.F., M.N.R.A.S. 145, 163.

Hatzidimitriou, D., Cannon, R.D., Hawkins, M.R.S., 1993, M.N.R.A.S. $261,873$.

Herbst, T.M., Beckwith, S.V.W., Forrest, W.J., Pipher, J.L., 1993, A.J. 105, 956.

Jorissen, A., Mayor, M., 1992, A.A. $260,115$.

Latham, D.W., 1992, IAU Coll. 135, 110.

Latham, D.W., Mazeh, T., Stefanik, R.P., Davis, R.J., Carney, B.W., Krymolowski, Y., Laird, J.B., Torres, G., Morse, J.A., 1992, A.J. 104, 774.

Mateo, M., Olzewski, E.W., Pryor, C., Welch, D.L., Fischer, P., 1993, A.J. $105,510$.

May, J., Bronfman, L., Alvarez, H., Murphy, D.C., Thaddeus, P., 1993, A.A. Sup. 99, 105.

Mehringer, D.M., Yusef-Zadeh, F., Palmer, P., Goss, W.M., 1992, Ap.J. 401, 168.

Mermilliod, J.C., Rosvick, J.M., Duquennoy, A., Mayor, M., 1992, A.A. 265, 526.

Minniti, D., White, S.D.M., Olzewski, E.W., Hill, J.M., 1992, Ap.J. 393, L47.

Morrell, N., Abt, H.A., 1992, Ap.J. 393, 666.

Morrison, H.L., 1993, A.J. 105, 539.

Murdoch, K., Hearnshaw, J.B., 1993, Ap.J., in press.

Prosser, C.F., 1993, A.J. 105, 1441.

Soderblom, D.R., Stauffer, J.R., Hudon, J.D., Jones, B.J., 1993, Ap.J.Sup. 85, 315.

Suntzeff, N.B., Schommer, R.A., Olzewski, E.W., Walker, A.R., 1992, A.J. 104, 1743.

Vassiliadis, E., Meatheringham, S.J., Dopita, M.A., 1992, Ap.J. 394, 489. 